Les transferts d'activité entre le secteur stationnaire et les soins ambulatoires sont fréquents. Les pressions économiques ainsi que le nouveau financement hospitalier sont susceptibles de générer des mouvements encore plus accentués entre les acteurs du système de santé. Après un premier état des lieux sous l'ancien régime, marqué par la coexistence de multiples systèmes de rétribution des hôpitaux et publié il y un an, les auteurs ont maintenant pu analyser l'évolution des habitudes après l'introduction de SwissDRG sur la base des données d'un grand assureur et nous présentent leurs résultats dans cet article. S'il n'y a pas de changements massifs, on peut néanmoins déjà observer certaines tendances.

Une étude plus détaillée sera effectuée secondairement, après l'obtention des chiffres officiels de la statistique des hôpitaux émis par l'OFS et en recourant aux informations disponibles chez New Index au sujet des médecins en pratique libre.

\title{
Effets de SwissDRG sur les soins ambulatoires*
}

\author{
Michael Lobsiger ${ }^{a}$, \\ Timo Tondelli ${ }^{b}$, \\ Wolfram Kägi ${ }^{a}$, \\ Stefan Felder ${ }^{b}$ \\ a B,S,S. Conseil économique, \\ Bâle \\ b Département «Health \\ Economics», WWZ, \\ Université de Bâle
}

\footnotetext{
* Une version détaillée de ce rapport intermédiaire effectué sur mandat de la FMH et de $\mathrm{H}+$ dans le cadre de la recherche concomitante en rapport avec l'introduction de SwissDRG est disponible sous www.fmh.ch $\rightarrow$ Tarifs hospitaliers $\rightarrow$ Recherche concomitante.

** Nous remercions Helsana Assurance-maladie SA pour les données mises à disposition ainsi que Mathias Früh d'Helsana Assurancemaladie SA pour son aide à la préparation des données.
}

\section{Correspondance:}

FMH / Département Tarifs et économie de la santé pour les médecins hospitaliers

Froburgstrasse 15

CH-4600 Olten

Tél. 0313591111

Fax 0313591112

\begin{abstract}
Enoncé du problème
L'analyse longitudinale a pour but de vérifier si l'introduction de la rémunération au forfait par cas des traitements stationnaires aigus (SwissDRG) a une influence sur la répartition du travail entre les secteurs stationnaire et ambulatoire, notamment sur les prestations (individuelles) fournies en amont ou en aval d'un traitement stationnaire. Servent ici d'indicateurs, le nombre de consultations ambulatoires données au cours d'une période déterminée avant et après le traitement stationnaire ainsi que les points tarifaires facturés à cet égard. La figure 1 offre une représentation graphique de la situation. La période de temps examinée varie entre 5,10 et 20 jours. En outre, une différenciation entre traitement ambulatoire à l'hôpital et en cabinet médical ainsi qu'entre médecin de famille et spécialiste est possible.
\end{abstract}

\section{Données et méthode}

La présente analyse se fonde sur les données relatives à la demande de prestations médicales de près de 68000 assurés de Helsana Assurance-maladie, qui ont reçu des soins stationnaires au moins une fois au $2^{\mathrm{e}}$ trimestre en 2010, 2011 ou $2012^{\star \star}$. En revanche, la demande de prestations ambulatoires par des assurés sans séjour stationnaire n'a pas été examinée.

Pour identifier l'effet de la rémunération au forfait par cas sur la demande de prestations ambulatoires avant ou après un traitement hospitalier, il convient de prendre en considération le fait que la rémunération des soins stationnaires aigus différait de canton à canton avant l'introduction de SwissDRG au $1^{\text {er }}$ janvier 2012. Dans toute la Suisse orientale et dans le canton de Fribourg, on appliquait une tarification des processus et prestations (PLT). Dans les deux Bâles, le canton de Soleure et celui de Lucerne, les hôpitaux de soins aigus étaient rémunérés selon des forfaits journaliers (FJ) pour les coûts de traitement des patients. Le canton d'Argovie représentait un cas particulier. En effet, alors que l'Hôpital cantonal d'Aarau avait recours, comme base de rémunération, à un modèle de cheminement intégré du patient (MCIP) qu'il avait lui-même développé, dans tous les autres hôpitaux publics ou subventionnés par les pouvoirs publics en Argovie, on facturait selon des forfaits par division (ABP). Il est intéressant de noter que, déjà avant l'introduction de SwissDRG, une grande partie des cantons de Suisse romande, centrale et méridionale utilisaient des forfaits par cas liés au diagnostic (AP-DRG). Dans ce contexte, on est quasiment face à une situation dans laquelle les cantons AP-DRG serviraient de groupe de contrôle, en utilisant une méthode fondée sur les «écarts dans les différences» (difference in difference). Celle-ci consiste à comparer les différences entre les régions AP-DRG et les régions disposant d'un autre système de rémunération, avant et après l'introduction de SwissDRG. Ainsi, il est possible de tenir compte d'une tendance générale (indépendante du système de rémunération) à une plus grande quantité de prestations ambulatoires et des différences non observables entre les systèmes de rémunération (pour autant que ces différences restent constantes sur la durée). Par ailleurs, une régression multivariée nous permet de contrôler toute une série de facteurs influents tels que les caractéristiques des patients (entre autres, fac-

\section{Figure 1}

Les prestations dans le domaine ambulatoire en amont et en aval.

\begin{tabular}{|c|c|c|}
\hline $\begin{array}{l}\text { Traitement en } \\
\text { amont } \\
\text { (ambulatoire) }\end{array}$ & $\begin{array}{c}\text { Séjour à } \\
\text { l'hôpital } \\
\text { (stationnaire) }\end{array}$ & $\begin{array}{r}\text { Traitement en } \\
\text { aval } \\
\text { (ambulatoire) }\end{array}$ \\
\hline & urée du traitem & \\
\hline
\end{tabular}




\section{Tableau 1}

Evolution des consultations en amont et en aval en comparaison avec SwissDRG (période: 5 , 10 et 20 jours).

\begin{tabular}{llll} 
& \multicolumn{4}{c}{ Ambulatoire en amont et en aval } \\
Période & $\mathbf{5}$ jours & $\mathbf{1 0}$ jours & $\mathbf{2 0}$ jours \\
\hline$\varnothing$ SwissDRG & $\mathbf{1 , 2 3}$ & $\mathbf{2 , 3 3}$ & 3,98 \\
\hline PLT & $0,077^{*}$ & $0,157^{* *}$ & $0,229^{* *}$ \\
\hline FJ & $0,106^{* * *}$ & $0,173^{* * *}$ & $0,255^{* * *}$
\end{tabular}

Remarque: ${ }^{*} / * * / * * *$ significatif au niveau $10 \% / 5 \% / 1 \%$.

Systèmes tarifaires: PLT: tarification des processus et prestations, FJ: forfaits journaliers.

teurs sociodémographiques, situation contractuelle, degré de gravité, PCG) ainsi que celles des hôpitaux (type d'hôpital, case-mix des patients traités, etc.).

\section{Résultats: nombre de consultations}

Le tableau 1 présente le nombre de consultations dans le secteur ambulatoire avant et après traitement stationnaire, pour les périodes de 5, 10 et 20 jours. A titre de comparaison, la première ligne indique la moyenne des consultations en amont et en aval pour le système tarifaire SwissDRG.

Les résultats montrent qu'après l'introduction de SwissDRG, les consultations ambulatoires ont augmenté dans les régions qui avaient recours aux systèmes PLT et FJ, en comparaison avec les cantons utilisant le système AP-DRG. Pour la période de 20 jours, l'augmentation est respectivement de $6.4 \%$ (PLT) et de $5.6 \%$ (FJ). On peut constater une hausse des consultations pour toutes les périodes; comme on pouvait s'y attendre, les effets sont moindres sur les périodes plus courtes.

La distinction entre avant et après le traitement stationnaire met en évidence une plus forte augmentation des consultations ambulatoires en amont du séjour stationnaire dans les régions ayant recours aux systèmes PLT et FJ, après l'introduction de SwissDRG, alors qu'on constate une plus forte augmenta-

\section{Tableau 2}

Evolution des points tarifaires en amont et en aval en comparaison avec SwissDRG (période: 5, 10 et 20 jours).

\section{Ambulatoire en amont et en aval}

\begin{tabular}{|c|c|c|c|}
\hline \multirow[b]{2}{*}{ Période } & \multicolumn{3}{|c|}{ Ambulatoire en amont et en aval } \\
\hline & 5 jours & 10 jours & 20 jours \\
\hline \multicolumn{4}{|c|}{ Points tarifaires TARMED } \\
\hline$\varnothing$ SwissDRG & 207,4 & 392,8 & 677,9 \\
\hline PLT & 9,81 & 15,09 & 23,94 \\
\hline TP & $16,04^{*}$ & 14,30 & 18,62 \\
\hline \multicolumn{4}{|c|}{ Points tarifaires TARMED + points des analyses de laboratoire } \\
\hline$\varnothing$ SwissDRG & 244,5 & 460,1 & 788,2 \\
\hline PLT & 15,17 & 25,65 & 43,01 \\
\hline FJ & $19,36^{*}$ & 17,44 & 25,14 \\
\hline
\end{tabular}

Remarque: * /** / *** significatif au niveau $10 \%$ / $5 \%$ / $1 \%$

Systèmes tarifaires: PLT: tarification des processus et prestations, F): forfaits journaliers. tion des consultations ambulatoires après le séjour hospitalier dans les régions qui facturaient auparavant avec des forfaits journaliers.

De plus, les résultats montrent que c'est en premier lieu le secteur de la pratique privée ambulatoire qui est concerné par les transferts. Par contre, il n'y a pas d'effets significatifs visibles pour le domaine ambulatoire hospitalier. Ces résultats se fondent sur des évaluations effectuées avec une période de 20 jours.

Pour les médecins de famille, il n'y a guère d'éléments indiquant une hausse des consultations après l'introduction de SwissDRG. Mais chez les spécialistes, les régions facturant anciennement avec les systèmes PLT et FJ enregistrent, par rapport au système AP-DRG, une forte hausse des consultations avant et après un traitement stationnaire.

\section{Nombre de points tarifaires facturés}

Le tableau 2 résume les résultats concernant les points tarifaires facturés pour les périodes de 5,10 et 20 jours. A titre de comparaison, la ligne ø SwissDRG donne la moyenne des points tarifaires facturés avec le système tarifaire SwissDRG. L'analyse distingue entre points TARMED et point TARMED + points des analyses de laboratoire.

Les coefficients évalués montrent une augmentation des coûts après l'introduction de SwissDRG. Toutefois, les effets ne sont pas significatifs, hormis une exception (FJ pour la période de 5 jours). Pour la période de 20 jours, l'augmentation est respectivement de $3.5 \%$ (PLT) et de $2.8 \%$ (FJ) pour les points TARMED et de $5.5 \%$ (PLT) et $3.2 \%$ (FJ) pour les points TARMED + points des analyses de laboratoire.

Pour la période de 20 jours, la distinction entre les domaines ambulatoires en amont et en aval ne change en principe rien à l'affirmation donnée plus haut. Les effets ne sont pas significatifs. En considération des deux autres périodes (5 et 10 jours), on n'observe un coefficient significativement positif que pour le système de rémunération $\mathrm{FJ}$ dans la période de 5 jours et les traitements en aval (points tarifaires TARMED + points des analyses de laboratoire) et pour le système de rémunération PLT dans la période de 10 jours et les traitements en aval (points tarifaires TARMED + points des analyses de laboratoire).

De plus, les résultats montrent que c'est le domaine de prestations ambulatoire en cabinet médical qui est avant tout concerné. En particulier dans les régions facturant anciennement avec un système de forfaits journaliers (FJ), on constate des hausses de coûts significatives après l'introduction de SwissDRG, tant pour l'ensemble que si l'on différencie entre amont et aval (période de 20 jours). Pour les régions qui utilisaient auparavant le système PLT, les coefficients évalués indiquent également une augmentation des coûts. Toutefois, les effets ne sont pas significatifs, sauf en ce qui concerne l'effet sur le secteur ambulatoire après traitement stationnaire. Dans 
le secteur ambulatoire hospitalier, on ne constate pas d'effets significatifs.

De (faibles) éléments indiquent une augmentation significative des points tarifaires facturés par le groupe des médecins de famille, dans les cantons où l'on facturait autrefois avec le système FJ. Cela vaut aussi bien pour le domaine ambulatoire en amont que pour celui en aval. Par contre, pour le groupe des médecins spécialistes, les effets montrent une hausse des coûts significative pour les régions qui utilisaient auparavant un système de forfaits journaliers (en amont et en aval) ainsi que pour celles utilisant autrefois un système PLT (en aval).

\section{Résumé}

Les effets de l'introduction de SwissDRG font l'objet d'une analyse longitudinale portant sur les transferts partiels de la prise en charge stationnaire vers les soins ambulatoires. Pour ce faire, un fichier de données complet de Helsana Assurance-maladie est à disposition. Pour l'analyse, une approche du type «écarts dans les différences» a été choisie. Il s'agit en fait de comparer les différences entre les régions (anciennement) avec AP-DRG et les régions disposant (autrefois) d'un autre système de rémunération, avant et après l'introduction de SwissDRG. L'observation se limite aux régions qui utilisaient les systèmes PLT et FJ.

Par rapport aux cantons utilisant le système APDRG, les consultations ambulatoires ont augmenté après l'introduction de SwissDRG, dans les régions qui avaient recours aux systèmes PLT et FJ. Pour les cantons qui, avant 2012, facturaient selon le système PLT, l'effet de SwissDRG est plus grand pour les consultations situées en amont que pour celles situées en aval. Et, pour les cantons qui, avant l'introduction de SwissDRG, avaient recours au système

tarifaire FJ, l'effet sur les domaines en amont et en aval est à peu près le même. Les effets portent principalement sur les prestations ambulatoires fournies en cabinet médical. Pour les spécialistes, on observe des effets significatifs; le nombre de consultations augmente plus fortement que dans les cantons qui utilisaient le système tarifaire AP-DRG avant l'introduction de SwissDRG.

Par rapport au système AP-DRG, on ne constate dans l'ensemble aucune augmentation significative des points tarifaires facturés en ambulatoire. Pour les traitements après un séjour hospitalier, seules les périodes de 5 jours (FJ et PLT) et de 10 jours (PLT, seulement TARMED + points des analyses de laboratoire) enregistrent des coefficients significativement positifs. Une analyse différenciée entre cabinets médicaux et secteur ambulatoire hospitalier montre que, dans la pratique privée ambulatoire, il y a une plus forte augmentation des points tarifaires facturés que dans le système AP-DRG. Dans le domaine ambulatoire hospitalier, on ne constate pas d'effet significatif. Des éléments indiquent une hausse des coûts chez les médecins de famille des cantons qui rémunéraient selon des forfaits journaliers. S'agissant des spécialistes, des éléments probants existent pour une augmentation des coûts dans le domaine en amont et en aval dans les cantons qui, avant 2012, facturaient selon des forfaits journaliers, ainsi que pour une augmentation des coûts dans le domaine en aval dans les cantons qui utilisaient le système tarifaire PLT avant l'introduction de SwissDRG.

Une prochaine étape consistera à évaluer les données de la statistique médicale des hôpitaux et de la statistique des hôpitaux (OFS) ainsi que les données de New Index. A cette occasion, l'accent sera mis sur l'aspect du transfert global de traitements du secteur stationnaire au secteur ambulatoire et en sens inverse.

\section{accéder au forum: www.bullmed.ch/forum/ \\ et scientifique ayant \\ trait au systeme de sante commentez les affirmations}

